\title{
Influência da aplicação de carboidrases \\ comerciais na extração e nas características \\ da polpa de medronho
}

\section{The influence of the application of commercial carbohydrases on the extraction and characteristics of strawberry tree fruit pulp}

\author{
Sofia Rosário Figueiredo", Ana Raquel Borges ${ }^{1}$, Marta Henriques ${ }^{1}$, Ivo Rodrigues²* (D) \\ ${ }^{1}$ Instituto Politécnico de Coimbra, Escola Superior Agrária de Coimbra, Departamento de Ciência e Tecnologia \\ Alimentar, Coimbra - Portugal \\ ${ }^{2}$ Centro de Investigação de Recursos Naturais, Ambiente e Sociedade (CERNAS), Instituto Politécnico de Coimbra, \\ Escola Superior Agrária de Coimbra, Coimbra - Portugal
}

${ }^{*}$ Corresponding Author: Ivo Rodrigues, Centro de Investigação de Recursos Naturais, Ambiente e Sociedade (CERNAS), Instituto Politécnico de Coimbra, Escola Superior Agrária de Coimbra, Campus, Bencanta, 3045-601, Coimbra - Portugal, e-mail: ivorod@esac.pt

Cite as: Figueiredo, S. R., Borges, A. R., Henriques, M., \& Rodrigues, I. (2021). The influence of the application of commercial carbohydrases on the extraction and characteristics of strawberry tree fruit pulp. Brazilian Journal of Food Technology, 24, e2020028. https://doi.org/10.1590/1981-6723.02820

\begin{abstract}
Resumo
Os medronhos são frutos com um alto teor de açúcares (40\%), antioxidantes e vitaminas, tais como vitamina $C$, $\beta$ caroteno, niacina, tocoferóis e ácidos orgânicos. No entanto, o rendimento de extração da polpa pelos métodos convencionais é baixo, correspondendo aproximadamente a $42 \%$. Este estudo teve como objetivo avaliar o potencial uso de duas carboidrases comerciais, Viscozyme ${ }^{\circledR}$ L e Pectinex ${ }^{\circledR}$ Ultra SP-L, na extração de polpa de medronho e a sua influência nas características físico-químicas e reológicas da polpa extraída. As carboidrases foram testadas sob diferentes temperaturas $\left(20^{\circ} \mathrm{C}\right.$ a $25^{\circ} \mathrm{C}$ e $\left.45^{\circ} \mathrm{C}\right)$, concentrações $(1,2$ e $4 \mu \mathrm{L} / \mathrm{g}$ de fruto macerado) e tempos de reação (30 e $60 \mathrm{~min}$ ). As mesmas condições foram aplicadas a amostras-controle sem adição de enzima. Foram determinados os rendimentos de extração ( $\mathrm{g}$ de polpa extraída/ $100 \mathrm{~g}$ de fruto macerado), viscosidade aparente (a 20,0 $\pm 0,1^{\circ} \mathrm{C}$ ), cor, acidez titulável, pH, sólidos solúveis ('Brix) e sólidos totais das polpas obtidas. A polpa com menor viscosidade aparente $(0,88 \pm 0,38$ Pa.s $)$ foi obtida pela aplicação da Viscozyme ${ }^{\circledR} \mathrm{L}$ a $4 \mu \mathrm{L} / \mathrm{g}, 45^{\circ} \mathrm{C}$, após 30 minutos de incubação. A Pectinex ${ }^{\circledR}$ Ultra SP-L foi responsável pelo rendimento de extração de polpa mais elevado $(54,9 \pm 1,1 \%)$ após $30 \mathrm{~min}$, quando aplicada a $2 \mu \mathrm{L} / \mathrm{g}$ e $45^{\circ} \mathrm{C}$. Foi também responsável pelo maior teor de sólidos solúveis $\left(27,3 \pm 0,1^{\circ} \mathrm{Brix}\right)$, à temperatura ambiente $\left(20^{\circ} \mathrm{C}\right.$ a $\left.25^{\circ} \mathrm{C}\right)$ após 60 minutos para a concentração 4 $\mu \mathrm{L} / \mathrm{g}$. Não foram encontradas diferenças significativas $(p>0,05)$ para as demais características físico-químicas das polpas, entre as condições testadas. A metodologia de extração de polpa assistida por carboidrases forneceu resultados promissores para a diversificação de produtos de valor acrescentado derivados do medronho, com elevado potencial de utilização na indústria alimentar (bebidas de fruta, doces e geleias, gelados, entre outros).
\end{abstract}

Palavras-chave: Arbutus unedo L.; Rendimento de extração; Viscozyme ${ }^{\circledR}$ L; Pectinex ${ }^{\circledR}$ Ultra SP-L; Viscosidade; Sólidos solúveis ( ${ }^{\circ}$ Brix). 


\begin{abstract}
Arbutus unedo's fruits have a high content of sugars (40\%), antioxidants, vitamins such as vitamin $C, \beta$-carotene, niacin, tocopherols and organic acids. However, pulp extraction yield by conventional methods is low corresponding approximately to $42 \%$. This study aims to evaluate the potential use of two commercial carbohydrases, Viscozyme ${ }^{\circledR}$ L and Pectinex ${ }^{\circledR}$ Ultra SP-L for pulp extraction from strawberry tree fruit and their influence on the physicochemical and rheological characteristics of the recovered pulp. Carbohydrases enzymes were tested under different temperatures $\left(20^{\circ} \mathrm{C}\right.$ to $25^{\circ} \mathrm{C}$ and $\left.45^{\circ} \mathrm{C}\right)$, enzime concentration $(1,2$ and $4 \mu \mathrm{L} / \mathrm{g}$ of macerated fruit) and reaction time (30 and $60 \mathrm{~min}$ ). The same conditions were applied for control samples without enzyme addition. Pulp extraction yield ( $\mathrm{g}$ of extracted pulp $/ 100 \mathrm{~g}$ macerated fruit), apparent viscosity (at $20 \pm 0.1{ }^{\circ} \mathrm{C}$ ), color, titratable acidity, $\mathrm{pH}$, soluble solids ( $\left.{ }^{\circ} \mathrm{Brix}\right)$, and total solids were assessed. The pulp with lower apparent viscosity $\left(0.88 \pm 0.38\right.$ Pa.s) was obtained by applying Viscozyme ${ }^{\circledR} \mathrm{L}$ at $4 \mu \mathrm{L} / \mathrm{g}, 45^{\circ} \mathrm{C}$ after 30 minutes of incubation. Pectinex ${ }^{\circledR}$ Ultra SP-L was responsible for the highest pulp extraction yield (54.9 $\pm 1.1 \%$ ) after $30 \mathrm{~min}$, at $2 \mu \mathrm{L} / \mathrm{g}$ and $45^{\circ} \mathrm{C}$. It was also responsible for the highest soluble solids content $\left(27.3 \pm 0.1^{\circ} \mathrm{Brix}\right)$ at room temperature $\left(20^{\circ} \mathrm{C}\right.$ to $\left.25^{\circ} \mathrm{C}\right)$, after $60 \mathrm{~min}$ at $4 \mu \mathrm{L} / \mathrm{g}$. No significant differences $(p>0.05)$ were found for the remaining physicochemical characteristics of the extracted pulps under the tested conditions. Carbohydrases-assisted extraction methodology provided promising results to increase the portfolio of added value products from strawberry tree fruit with high potential application in the food industry (fruit beverages, jams, ice creams, among others).
\end{abstract}

Keywords: Arbutus unedo L.; Pulp extraction yield; Viscozyme ${ }^{\circledR}$ L; Pectinex ${ }^{\circledR}$ Ultra SP-L; Viscosity; Soluble solids ('Brix).

\title{
1 Introdução
}

O medronheiro (Arbutus unedo L.) é uma espécie autóctone mediterrânea e da Europa Ocidental, sendo também possível encontrá-la no oeste da França, na Irlanda e em vários países do norte da África, onde cresce espontaneamente em solos drenados e zonas rochosas (Torres et al., 2002; Gomes \& Canhoto, 2009). Em Portugal, é considerada uma espécie NUC (neglected or underutilized crop). Esta classificação atribui ao medronheiro interesse agrícola e potencial agroindustrial, em especial para as populações residentes nos locais de onde é nativo, mas que, por razões diversas, não é ainda convenientemente explorado (Gomes et al., 2010; Global Facilitation Unit for Underutilized Species, 2013).

A sua predominância em Portugal continental é verificada ao sul do Rio Tejo, essencialmente no Algarve, nas Serras de Monchique e do Caldeirão. Não obstante, está também difundido no restante território, como é o caso de Trás-os-Montes (Pedro, 1994), onde se desenvolve em associação com comunidades arbustivas (Prada \& Arizpe, 2008). Dada a sua composição rica em fibra e em compostos bioativos, o medronheiro pode ainda caracterizar-se como espécie multifuncional, podendo ser utilizado em fármacos, cosméticos, na alimentação e na saúde (Global Facilitation Unit for Underutilized Species, 2013).

O fruto, denominado de "medronho", para além de uma porcentagem elevada de açúcares ( $42 \%$ a $52 \%$, na base seca), é uma boa fonte de minerais (Özcan \& Haciseferoğullari, 2007), vitaminas C e E, carotenoides e antioxidantes. A presença de compostos fenólicos, tais como antocianinas, outros flavonoides, derivados do ácido gálico e taninos, é responsável por muitas das funções biológicas atribuídas ao consumo de medronho (Alarcão-e-Silva et al., 2001; Mendes et al., 2011; Oliveira et al., 2011; Malheiro et al., 2012; Morales et al., 2013). Além do consumo em fresco, estes frutos podem ser transformados em compotas, geleias, ou dar origem a bebidas alcoólicas fermentadas e destiladas, com elevado teor alcoólico ( $45 \%$ a 50\% de etanol, v/v) (Santo et al., 2012). Atualmente, esta é a via de exploração com maior expressão econômica (Gomes et al., 2010).

Outras utilizações têm sido exploradas para a utilização do medronho, nomeadamente para incorporação em iogurtes, produtos de confeitaria ou cereais de café da manhã (Alarcão-e-Silva et al., 2001), sob a forma de polpa, frutos frescos, secos ou desidratados. Esse fruto possui ainda elevado potencial na produção de 
corantes alimentares naturais, dada a composição rica em $\beta$-caroteno e antocianinas (Molina et al., 2011). Guerreiro et al. (2013) concluíram que, durante pelo menos 15 dias, a cor e o sabor dos medronhos frescos armazenados a $0{ }^{\circ} \mathrm{C}$ não sofrem alteração significativa, o que constitui uma característica determinante na sua comercialização em fresco. Tal fato permite contrariar a rápida deterioração do fruto em estado de maturação avançado, contribuindo desta forma para o interesse frutícola da espécie. Por estas razões, o cultivo do medronheiro tem despertado crescente interesse em zonas com solos degradados, condições de estresse hídrico elevado e elevado risco de incêndios, pois é uma espécie autóctone, rústica, bem adaptada e resiliente, que pode ser utilizada em faixas de proteção (linhas de água e na defesa da floresta contra incêndios).

Este trabalho enquadra-se na valorização e diversificação econômica do medronho e apresenta um estudo contributivo para o aumento do rendimento da extração de polpa através da ação de duas enzimas comerciais, a Viscozyme ${ }^{\circledR}$ L e a Pectinex ${ }^{\circledR}$ Ultra SP-L. A natureza multicomponente da Viscozyme ${ }^{\circledR}$ L tem particular utilidade para o processamento de matérias-primas vegetais destinadas à produção de álcool, cerveja, amido e indústrias relacionadas. A sua capacidade para degradar os polissacarídeos não amiláceos pode melhorar a disponibilidade do amido na fermentação, reduzir a viscosidade e, portanto, melhorar os rendimentos de extração (Novozymes, 2002, 2012). Quanto à Pectinex ${ }^{\circledR}$ Ultra SP-L, é um complexo enzimático com elevada atividade pectolítica, contendo alguma atividade hemicelulásica, sendo a sua composição especialmente desenvolvida para processamento de polpa de frutas e vegetais, otimizando a capacidade de separação sólido/líquido e o rendimento em suco (Novo Nordisk BioChem, 1998; Novozymes, 2006).

A utilização, quer da Viscozyme ${ }^{\circledR}$ L, quer da Pectinex ${ }^{\circledR}$ Ultra SP-L, tem sido estudada na extração de sucos, polpas, óleos essenciais etc., mas não na extração de polpa de medronho, tanto quanto é do nosso conhecimento. Mais recentemente, a Pectinex ${ }^{\circledR}$ Ultra SP-L foi testada com sucesso na extração de sucos de mirtilo (Siddiq et al., 2018) e de groselha (Loan et al., 2017), de purês de graviola (Chang et al., 2018) e de abacaxi (Wong et al., 2015). A utilização da Viscozyme ${ }^{\circledR}$ L, conjugada ou não com a Pectinex ${ }^{\circledR}$ Ultra SPL, foi estudada na extração de suco de amora (Nguyen \& Nguyen, 2018), na extração de óleos essenciais de cardamomo, de oleoresinas (Baby \& Ranganathan, 2016a), de capsaisinoides da pimenta verde (Baby \& Ranganathan, 2016b) e ainda para incremento do teor de proteína em bagaços de colza (Rodrigues et al., 2014).

Neste estudo, pretende-se avaliar a influência da utilização destes complexos enzimáticos na extração e nas propriedades físico-químicas da polpa de medronho, além de otimizar as condições de temperatura, concentração de enzima e tempo da reação. Neste sentido, será estudada a viabilidade da produção de uma nova categoria de produtos alimentares derivados do medronho, de características composicionais idênticas às do fruto em natureza, isentos de sementes e esclereídeos.

\section{Material e métodos}

\subsection{Materiais}

Os frutos de Arbutus unedo L., com cor entre o laranja e o vermelho, foram colhidos na zona Centro de Portugal, em outubro de 2016. Procedeu-se à maceração dos frutos $(\cong 8 \mathrm{~kg})$ e ao embalamento da pasta em porções de aproximadamente $600 \mathrm{~g}$, que foram acondicionadas em sacos de vácuo e conservadas em câmara de congelação a $-20^{\circ} \mathrm{C}$ até utilização.

A Viscozyme ${ }^{\circledR}$ L (Sigma-Aldrich, Alemanha) é um complexo de multienzimas degradativas dos hidratos de carbono (arabanase, celulase, $\beta$-glucanase, hemicelulase e xilanase) com maior atividade enzimática para a $\beta$-glucanase. A sua atividade enzimática é de $100 \mathrm{FBG}$ (unidades de $\beta$-glucanase fúngica) por mililitro. Segundo o fornecedor, as condições ótimas para maximizar a atividade deste complexo são: $\mathrm{pH}=3,3$ a 5,5, temperatura entre $40^{\circ} \mathrm{C}$ e $50^{\circ} \mathrm{C}$, na proporção de 0,05 a $0,1 \%$ (v/m) (Novozymes, 2002, 2012). 
A Pectinex ${ }^{\circledR}$ Ultra SP-L (Novozymes, Dinamarca) é um complexo enzimático com elevada atividade pectolítica, contendo alguma atividade hemicelulásica. A sua atividade enzimática é de 26.000 PG (unidades de poligalacturonase) por mililitro. As condições recomendadas para maximizar a sua atividade enzimática são $\mathrm{pH}=3,5$, à temperatura de $35^{\circ} \mathrm{C}$ e dosagem de $200 \mu \mathrm{L} / \mathrm{kg}$ (Novo Nordisk BioChem, 1998; Novozymes, 2006).

\subsection{Tratamento enzimático}

Imediatamente antes do tratamento enzimático, foram descongeladas duas amostras de pasta macerada, uma à temperatura ambiente $\left(20^{\circ} \mathrm{C}\right.$ a $\left.25{ }^{\circ} \mathrm{C}\right)$ e outra a $45{ }^{\circ} \mathrm{C}$, em estufa. Cada amostra descongelada foi dividida em oito porções, nas quais foram aplicadas soluções aquosas de Viscozyme ${ }^{\circledR}$ L e de Pectinex ${ }^{\circledR}$ Ultra_SP-L, com concentrações de $0 \mu \mathrm{L} / \mathrm{g}$ (controle), 1,2 e $4 \mu \mathrm{L} / \mathrm{g}$ de pasta macerada, baseadas nas recomendações do fornecedor e na atividade enzimática de cada complexo. Após a adição das enzimas, as amostras foram colocadas às mesmas temperaturas aplicadas durante a descongelação. Os tempos de reação foram definidos com base em ensaios preliminares que indicaram a estabilização da concentração de sólidos solúveis a partir dos 30 minutos de reação. No caso da pasta descongelada a $20^{\circ} \mathrm{C}$ a $25{ }^{\circ} \mathrm{C}$, por se tratar de uma gama de temperaturas inferior às recomendadas pelos fornecedores para as enzimas em causa, foi também testada a incubação durante 60 minutos.

O estudo nestas condições teve como principal objetivo a avaliação da performance do tratamento enzimático à temperatura ambiente, evitando gastos energéticos adicionais durante o processamento. Para os ensaios a $45{ }^{\circ} \mathrm{C}$, o tempo de incubação foi apenas de 30 minutos, uma vez que se encontra no intervalo de temperatura recomendado para a Viscosyme ${ }^{\circledR}$ L e um pouco superior à indicada para a Pectinex ${ }^{\circledR}$ Ultra_SPL. Após a incubação, as amostras foram colocadas no micro-ondas (Samsung, Malásia) e sujeitas à potência de $750 \mathrm{~W}$ durante $15 \mathrm{~s}$, atingindo cerca de $85^{\circ} \mathrm{C}$, garantindo a sua pasteurização e consequente desnaturação enzimática. Em seguida, foram arrefecidos num banho de água gelada, até atingirem a temperatura ambiente.

Com vista à separação eficiente da polpa do medronho dos restantes componentes sólidos do fruto (as sementes e os esclereídeos), a pasta já arrefecida foi submetida à prensagem através de tela de tecido TV ( $100 \%$ poliamida), com poro 0,1 a $0,2 \mathrm{~mm}$ (Freire e Prego Ltda., Portugal), vulgarmente utilizado na produção de queijo.

$\mathrm{Na}$ avaliação da ação das diferentes enzimas e condições de tratamento sobre a pasta macerada de medronho, foram determinados os rendimentos de extração de polpa e expressos em porcentagem. Estes foram obtidos através da razão entre a quantidade de polpa obtida resultante da prensagem e a massa das amostras de pasta macerada de medronho para cada tratamento, submetidas ou não (controle) a tratamento enzimático.

\subsection{Análises físico-químicas}

Foram realizadas análises físico-químicas para caracterização da pasta macerada e das polpas, nomeadamente: viscosidade aparente $\left(\mathrm{a} 20,0 \pm 0,1^{\circ} \mathrm{C}\right)$, cor $\left(\mathrm{L}^{*} \mathrm{a}^{*} \mathrm{~b}^{*}\right)$, acidez titulável (g ácido cítrico/100 $\mathrm{g}$ ), $\mathrm{pH}$, sólidos solúveis ( $\left.{ }^{\circ} \mathrm{Brix}\right)$ e sólidos totais.

A viscosidade aparente (Pa.s) das polpas obtidas foi determinada utilizando o reômetro Rheostress 6000 (Haake, Thermo Scientific, Austrália). Os ensaios foram realizados imediatamente após a extração da polpa, a $20,0 \pm 0,1^{\circ} \mathrm{C}$, com taxa de cisalhamento de $10 \mathrm{~s}^{-1}$ mantida durante $20 \mathrm{~s}$.

A cor foi medida diretamente nas amostras através do colorímetro Minolta CR-200b (Japão), no sistema CIELab e expressa em L*a*b*, em que $L^{*}$ representa a luminosidade e varia de 0 a 100 (preto a branco); $a^{*}$ representa as cores entre o verde (valores negativos) e o vermelho (valores positivos), e o $b^{*}$ representa as cores entre o azul (valores negativos) e o amarelo (valores positivos). 
Para a determinação do pH, foi utilizado o potenciômetro Crison micro pH 2002 (Espanha) com elétrodo de pH 5203. A acidez titulável (expressa em g de ácido cítrico por $100 \mathrm{~g}$ de amostra) foi avaliada por titulação com $\mathrm{NaOH} \mathrm{0,1} \mathrm{mol/L} \mathrm{até} \mathrm{pH} \mathrm{8,1} \mathrm{utilizando} \mathrm{cerca} \mathrm{de} 5 \mathrm{~g}$ de amostra diluída em $50 \mathrm{~mL}$ de água destilada.

O índice refratométrico, ou ${ }^{\circ}$ Brix, foi determinado através de um refratômetro digital HANNA HI 96801 (Portugal) (escala 0 a $85 \%$ ), de forma a quantificar a porcentagem de matéria solúvel presente nos frutos. A composição em sólidos totais foi determinada por secagem das amostras em estufa a $105^{\circ} \mathrm{C}$ até peso contante e os resultados foram expressos em porcentagem.

Todos os ensaios e análises foram realizados em triplicata Os resultados foram expressos como valores médios \pm desvio padrão. A análise estatística foi realizada por análise de variância (ANOVA) usando o software Statistica V.12.0 (StatSoft, Inc., 2013). Utilizou-se o teste Tukey HSD para verificar a existência de diferenças significativas entre os valores das médias dos parâmetros avaliados, com um nível de confiança de $95 \%$.

\section{Resultados e discussão}

A caracterização da pasta de medronho macerada apresenta-se na Tabela 1. Os resultados obtidos estão de acordo com a bibliografia consultada (Noronha, 2001; Cavaco, 2007; Özcan \& Haciseferoğullari, 2007; Celikel et al., 2008; Ruiz-Rodríguez et al., 2011; Sulusoglu et al., 2011).

Tabela 1. Caracterização da pasta macerada de medronho.

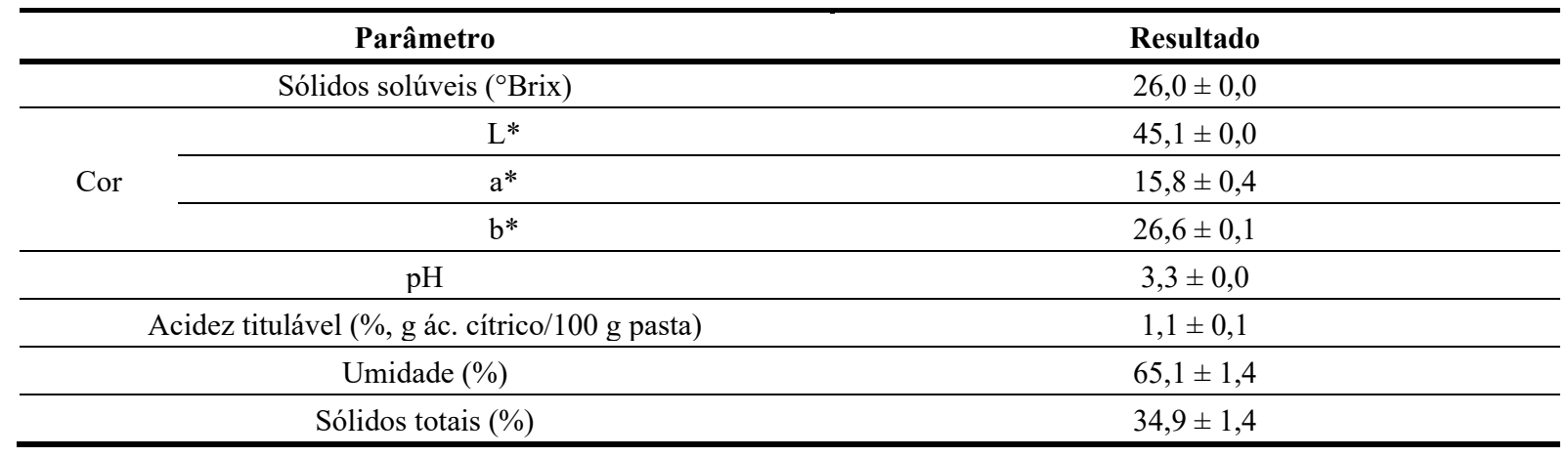

Os rendimentos de extração da polpa depois da aplicação do tratamento enzimático encontram-se na Figura 1.

No caso da Viscozyme ${ }^{\circledR}$ (Figura 1A), para a maior concentração testada e para a temperatura de $45^{\circ} \mathrm{C}$, que se encontra no intervalo de temperaturas ótimas recomendadas para este complexo enzimático, obtevese o melhor rendimento de extração $(46,9 \pm 0,5 \%)$. Ainda assim, este aumento não é significativo $(p>0,05)$ quando comparado com o controle. Também não foram encontradas diferenças significativas $(p>0,05)$ entre qualquer dos restantes tratamentos, indicando que a aplicação desta enzima não tem qualquer influência no rendimento de extração da polpa.

Quando aplicada a Pectinex ${ }^{\circledR}$ Ultra SP-L (Figura 1B), o rendimento de extração da polpa, para qualquer dos fatores testados, é sempre superior aos registrados para os controles $(p<0,05)$. Neste caso, o rendimento de extração mais elevado $(54,9 \pm 1,1 \%)$ foi obtido quando aplicada a concentração de $2 \mu \mathrm{L} / \mathrm{g}$, a $45^{\circ} \mathrm{C}$, durante 30 minutos. No entanto, este resultado é próximo $(p>0,05)$ ao alcançado no tratamento a $20-25^{\circ} \mathrm{C}$ durante 30 minutos $(52,8 \pm 1,1 \%)$, aplicando a concentração de enzima mais baixa $(1 \mu \mathrm{L} / \mathrm{g})$. Estas condições podem traduzir-se em custos mais baixos de processamento, já que implica a utilização de menor quantidade de enzima e ausência de gastos de energia durante o tempo de incubação. Quando comparada a utilização da Viscozyme ${ }^{\circledR}$ L (Figura 1B) com a da Pectinex ${ }^{\circledR}$ Ultra SP-L (Figura 1B), verifica-se que o rendimento de extração da polpa é sempre superior quando utilizada esta última. Pode-se afirmar que a Pectinex ${ }^{\circledR}$ Ultra SP-L é preferida à Viscozyme ${ }^{\circledR}$ L na extração de polpas de medronho, nas condições estudadas. 

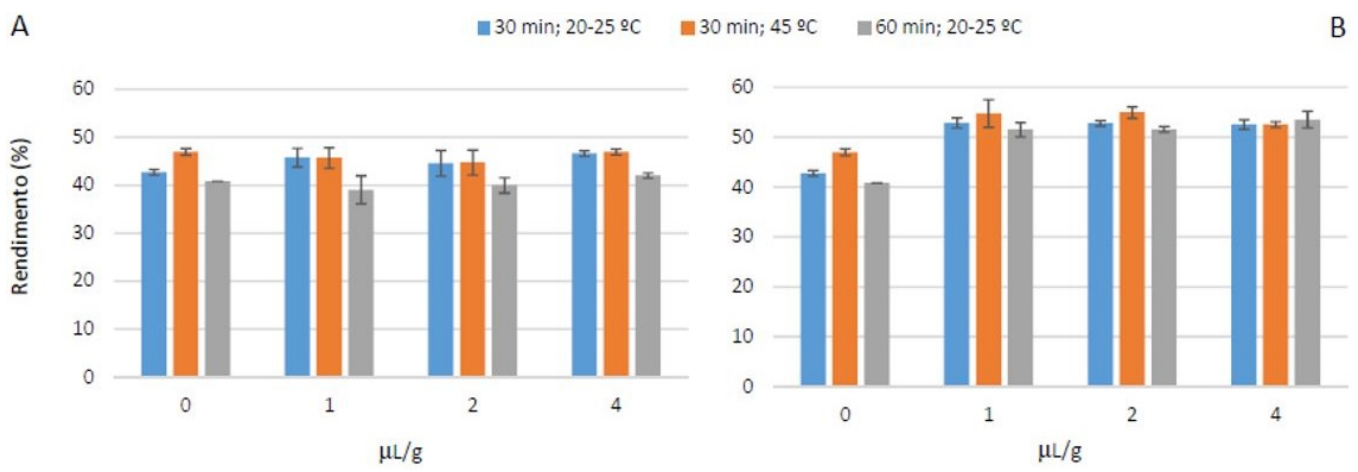

Figura 1. Rendimento de extração de polpa de medronho em função da concentração das enzimas Viscozyme ${ }^{\circledR}(\mathrm{A}) \mathrm{e}$ Pectinex ${ }^{\circledR}$ Ultra SP-L Ultra SP-L (B) em incubação de: 30 min a $20^{\circ} \mathrm{C}$ a $25^{\circ} \mathrm{C} ; 30$ min a $45^{\circ} \mathrm{C}$, e 60 min a $20^{\circ} \mathrm{C}$ a $25^{\circ} \mathrm{C}$.

Dadas as características das enzimas utilizadas e em concordância com a sua ação catalítica sobre os hidratos de carbono mais complexos em açúcares de baixo peso molecular, com influência direta no índice de refração, verifica-se um aumento significativo $(p<0,05)$ do ${ }^{\circ}$ Brix para a maioria das polpas produzidas, quer com a Viscozyme ${ }^{\circledR}$ L (Figura 2A), quer com a Pectinex ${ }^{\circledR}$ Ultra SP-L (Figura 2B), quando comparados com os valores dos controles $\left(26,0^{\circ} \mathrm{Brix}\right)$. Verifica-se também que, no caso da Viscozyme ${ }^{\circledR} \mathrm{L}$, o aumento da concentração da enzima é o fator responsável pelo aumento no teor de sólidos solúveis, justificado pela sua ação degradativa sobre o vasto conjunto de hidratos de carbono presentes em abundância no medronho (126 a $189 \mathrm{~g} / \mathrm{kg}$ fruto fresco) (Boussalah et al., 2018), e que os outros fatores (tempo e temperatura de incubação) não são determinantes neste parâmetro.

À semelhança do rendimento da extração da polpa, é também no tratamento com Pectinex ${ }^{\circledR}$ Ultra SP-L que se verificam os valores de ${ }^{\circ}$ Brix mais elevados $(27,3 \pm 0,1 \%)$, à temperatura ambiente, 60 minutos de incubação e para a concentração $4 \mu \mathrm{L} / \mathrm{g}$, sendo, neste caso, evidente a influência do aumento do tempo de incubação $(p<0,05)$. Quando comparada a performance da Viscozyme ${ }^{\circledR}$ L (Figura 2A) com a da Pectinex ${ }^{\circledR}$ Ultra SP-L (Figura 2B), verifica-se que esta última permite valores de ${ }^{\circ}$ Brix superiores e, por isso, será preferida quando se pretende a obtenção de polpas de medronho com maior teor de sólidos solúveis. Segundo Alarcão-eSilva et al. (2001), tal advém da sua elevada atividade pectolítica sobre a cadeia principal do ácido galacturônico das pectinas presentes na polpa de medronho em considerável concentração, 3,0 a 4,6\% (base seca).

Quando aplicadas estas enzimas na extração de sucos de amora, Nguyen \& Nguyen (2018) observaramse resultados semelhantes, obtendo maiores rendimentos de extração e sucos com ${ }^{\circ}$ Brix mais elevado, quando aplicada a Pectinex ${ }^{\circledR}$ Ultra SP-L. A utilização da mesma enzima proporcionou também o aumento do rendimento de extração de suco de mirtilo, mas sem alteração significativa do ${ }^{\circ}$ Brix (Siddiq et al., 2018), e ainda, quando aplicada na produção de polpa de graviola, apresentou teores de sólidos solúveis ( ${ }^{\circ}$ Brix) significativamente mais elevados (Chang et al., 2018).

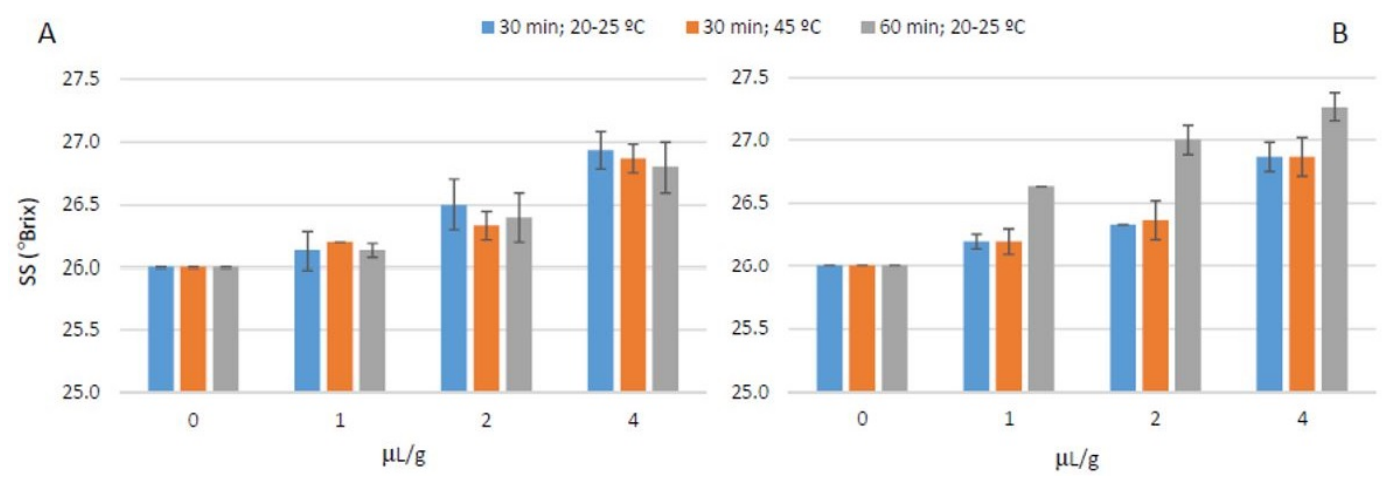

Figura 2. Sólidos solúveis (SS) na polpa de medronho em função da concentração das enzimas Viscozyme ${ }^{\circledR}$ (A) e Pectinex ${ }^{\circledR}$ Ultra SP-L (B) em incubação de 30 min a $2{ }^{\circ} \mathrm{C}$ a $25^{\circ} \mathrm{C} ; 30$ min a $45^{\circ} \mathrm{C}$, e 60 min a $20^{\circ} \mathrm{C}$ a $25^{\circ} \mathrm{C}$. 
A Figura 3 mostra a viscosidade aparente das polpas produzidas após a aplicação das enzimas. Em ambos os casos, verifica-se uma acentuada diminuição deste parâmetro relativamente ao controle $(p<0,05)$. $\mathrm{O}$ aumento da concentração da enzima e do tempo de incubação conduz a uma diminuição significativa $(p<0,05)$ da viscosidade da polpa, independentemente da enzima utilizada. É no tratamento da pasta de medronho com Viscozyme ${ }^{\circledR}$ L (Figura $\left.3 \mathrm{~A}\right)$, à temperatura ótima $\left(45^{\circ} \mathrm{C}\right)$ durante $30 \mathrm{~min}$, na concentração mais elevada $(4 \mu \mathrm{L} / \mathrm{g})$, que é observado o resultado mais baixo $\left(0,88 \pm 0,38\right.$ Pa.s). Para o caso da Pectinex ${ }^{\circledR}$ Ultra SP-L, a menor viscosidade aparente $\left(1,19 \pm 0,22\right.$ Pa.s) corresponde ao tratamento a $45{ }^{\circ} \mathrm{C}$, durante 30 minutos, também na concentração mais elevada $(4 \mu \mathrm{L} / \mathrm{g})$ (Figura 3B). A redução da viscosidade aparente promovida pela aplicação da Pectinex ${ }^{\circledR}$ Ultra SP-L foi também observada na produção de polpa de graviola (Chang et al., 2018) e de abacaxi (Wong et al., 2015).
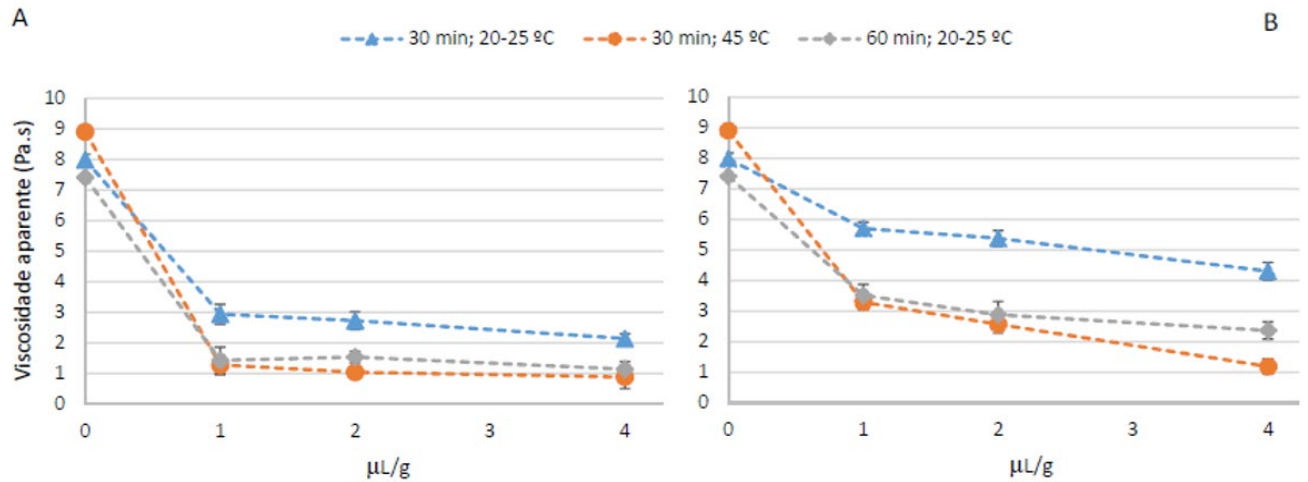

Figura 3. Viscosidade aparente da polpa de medronho em função da concentração das enzimas Viscozyme ${ }^{\circledR} \mathrm{L}$ (A) e Pectinex ${ }^{\circledR}$ Ultra SP-L (B) em incubação de $30 \min$ a $20^{\circ} \mathrm{C}$ a $25^{\circ} \mathrm{C} ; 30 \min$ a $45^{\circ} \mathrm{C}$, e 60 min a $20^{\circ} \mathrm{C}$ a $25^{\circ} \mathrm{C}$.

A cor das polpas de medronho, obtidas por ação do tratamento com Viscozyme ${ }^{\circledR}$ L e com a Pectinex ${ }^{\circledR}$ Ultra SP-L, é apresentada na Figura 4. Verifica-se que os parâmetros da cor $\left(\mathrm{L}^{*}, \mathrm{a}^{*} \mathrm{e} \mathrm{b}^{*}\right)$ das polpas de medronho não são influenciados $(p>0,05)$ por qualquer uma das enzimas utilizadas em qualquer condição de tratamento (tempo, temperatura ou concentração). No mesmo sentido, concluíram Chang et al. (2018) relativamente à cor da polpa de graviola, quando produzida com auxílio de Pectinex® Ultra SP-L. Já o suco de mirtilo produzido com o auxílio desta enzima sofreu uma alteração positiva nas características da cor (Siddiq et al., 2018). Desta forma, apesar de as polpas produzidas com auxílio destas enzimas manifestarem menor viscosidade, mantêm a cor semelhante à das polpas obtidas pela aplicação do processo de extração convencional, sem utilização de enzimas (controle). Comparando a cor das polpas com a cor da pasta macerada do fruto, verifica-se um ligeiro aumento na luminosidade e um aumento significativo $(p<0,05)$ no valor de $\mathrm{b}^{*}$, motivado pela remoção dos esclerídeos no processo de extração. Estas diferenças traduzem-se na alteração da percepção visual de um tom mais avermelhado para um tom laranja. Esta evidência associada ao aumento do ${ }^{\circ}$ Brix e do rendimento de extração tornam a aplicação destas enzimas ainda mais promissora na transformação deste fruto em polpa.

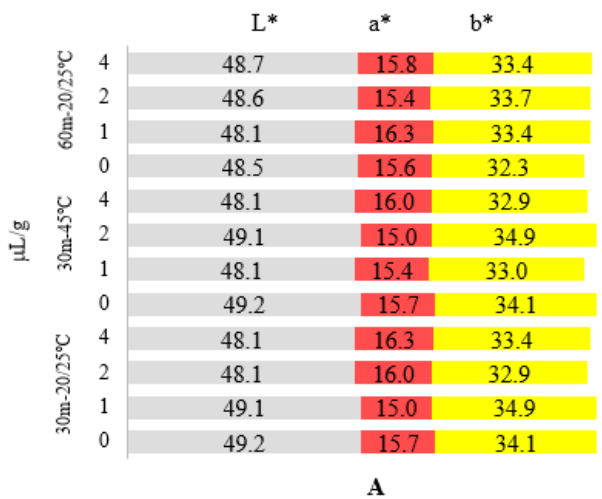

\begin{tabular}{|c|c|c|c|}
\hline & $\mathrm{L}^{*}$ & $a^{*}$ & $b^{*}$ \\
\hline 4 & 48.4 & 15.2 & 33.1 \\
\hline 2 & 48.9 & 15.9 & 32.8 \\
\hline 1 & 48.2 & 15.3 & 33.7 \\
\hline 0 & 48.3 & 15.7 & 32.2 \\
\hline 4 & 48.4 & 15.9 & 33.5 \\
\hline 2 & 48.4 & 15.5 & 33.8 \\
\hline 1 & 48.2 & 15.2 & 34.0 \\
\hline 0 & 48.2 & 15.6 & 33.1 \\
\hline 4 & 48.2 & 15.7 & 33.1 \\
\hline 2 & 48.3 & 15.1 & 32.5 \\
\hline 1 & 49.0 & 15.3 & 34.0 \\
\hline 0 & 48.1 & 15.8 & 32.8 \\
\hline
\end{tabular}

Figura 4. Cor (L*, a* e b*) da polpa de medronho com a aplicação de Viscozyme ${ }^{\circledR}$ L (A) e da Pectinex ${ }^{\circledR}$ Ultra SP-L (B), em função da concentração de enzima, tempo e temperatura de incubação. 
As polpas obtidas foram ainda caracterizadas quanto ao $\mathrm{pH}$, à acidez titulável e aos sólidos totais, como se pode verificar na Tabela 2. Quer a acidez titulável, quer o conteúdo em sólidos totais das polpas extraídas com aplicação das enzimas, não foram alterados significativamente em relação aos controles $(p>0,05)$. $\mathrm{O}$ $\mathrm{pH}$ das polpas apresenta um decréscimo, tanto maior quanto maior a concentração de enzima utilizada, na maioria dos casos, significativo ( $p<0,05$ ), quando comparado com o valor do respectivo controle nas mesmas condições de tempo e temperatura de incubação. Não se verificaram diferenças significativas $(p>0,05)$ entre os controles. Deve-se destacar que os valores de $\mathrm{pH}$ registrados encontram-se na gama de valores recomendados para os complexos enzimáticos utilizados, pelo que não se justificaria qualquer correção deste parâmetro operacional.

Tabela 2. pH, acidez titulável (AT) e sólidos totais (ST) das polpas de medronho, em função da enzima utilizada, do tempo e da temperatura de incubação, e da concentração de enzima.

\begin{tabular}{|c|c|c|c|c|c|}
\hline Enzima & Tempo $(\min ) /$ Temperatura $\left({ }^{\circ} \mathrm{C}\right)$ & $\begin{array}{c}\text { [Enzima] } \\
(\mu \mathrm{L} / \mathrm{g})\end{array}$ & pH & $\operatorname{AT}^{(1)}(\%)$ & $\mathrm{ST}^{(1)}(\%)$ \\
\hline \multirow{3}{*}{$\begin{array}{l}\frac{0}{0} \\
\stackrel{\Xi}{0} \\
0 \\
0 \\
0\end{array}$} & $30 / 20-25$ & 0 & $3,50 \pm 0,07^{\mathrm{a}, \mathrm{b}}$ & $1,0 \pm 0,2$ & $31,2 \pm 2,1$ \\
\hline & $30 / 45$ & 0 & $3,57 \pm 0,07^{\text {a }}$ & $1,0 \pm 0,2$ & $27,1 \pm 2,1$ \\
\hline & $60 / 20 / 25$ & 0 & $3,56 \pm 0,07^{\mathrm{a}}$ & $1,0 \pm 0,2$ & $31,1 \pm 2,1$ \\
\hline \multirow{9}{*}{ 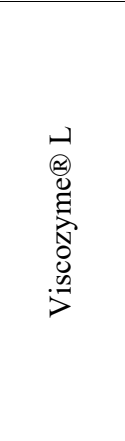 } & \multirow{3}{*}{$30-20 / 25$} & 1 & $3,36 \pm 0,02^{b, c}$ & $1,1 \pm 0,5$ & $25,5 \pm 1,1$ \\
\hline & & 2 & $3,29 \pm 0,01^{\mathrm{c}}$ & $1,1 \pm 0,0$ & $26,7 \pm 2,5$ \\
\hline & & 4 & $3,28 \pm 0,02^{c}$ & $1,1 \pm 0,3$ & $22,5 \pm 3,6$ \\
\hline & \multirow{3}{*}{$30-45$} & 1 & $3,54 \pm 0,11^{\mathrm{a}}$ & $1,1 \pm 0,3$ & $26,4 \pm 0,4$ \\
\hline & & 2 & $3,39 \pm 0,02^{\mathrm{a}, \mathrm{b}, \mathrm{c}}$ & $1,1 \pm 0,1$ & $26,5 \pm 0,2$ \\
\hline & & 4 & $3,32 \pm 0,02^{\mathrm{b}, \mathrm{c}}$ & $1,1 \pm 0,2$ & $26,8 \pm 0,2$ \\
\hline & \multirow{3}{*}{$60-20 / 25$} & 1 & $3,38 \pm 0,01^{\mathrm{a}, \mathrm{b}, \mathrm{c}}$ & $1,0 \pm 0,6$ & $28,2 \pm 0,4$ \\
\hline & & 2 & $3,30 \pm 0,01^{\mathrm{c}}$ & $1,1 \pm 1,0$ & $29,8 \pm 1,3$ \\
\hline & & 4 & $3,28 \pm 0,01^{\mathrm{c}}$ & $1,2 \pm 0,8$ & $27,5 \pm 0,1$ \\
\hline \multirow{9}{*}{ 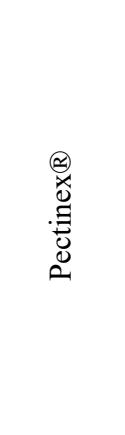 } & \multirow{3}{*}{$30-20 / 25$} & 1 & $3,35 \pm 0,07^{\mathrm{b}, \mathrm{c}}$ & $1,1 \pm 0,1$ & $28,8 \pm 0,9$ \\
\hline & & 2 & $3,30 \pm 0,02^{c}$ & $1,1 \pm 0,1$ & $29,3 \pm 0,1$ \\
\hline & & 4 & $3,29 \pm 0,02^{c}$ & $1,2 \pm 0,3$ & $29,1 \pm 0,6$ \\
\hline & \multirow{3}{*}{$30-45$} & 1 & $3,33 \pm 0,11^{\mathrm{b}, \mathrm{c}}$ & $1,1 \pm 0,3$ & $26,4 \pm 0,5$ \\
\hline & & 2 & $3,28 \pm 0,02^{\mathrm{c}}$ & $1,1 \pm 0,8$ & $26,1 \pm 0,3$ \\
\hline & & 4 & $3,28 \pm 0,02^{c}$ & $1,2 \pm 0,3$ & $26,4 \pm 0,5$ \\
\hline & \multirow{3}{*}{$60-20 / 25$} & 1 & $3,54 \pm 0,11^{\mathrm{a}}$ & $1,1 \pm 1,0$ & $32,6 \pm 1,2$ \\
\hline & & 2 & $3,39 \pm 0,02^{a, b, c}$ & $1,1 \pm 0,3$ & $33,1 \pm 1,1$ \\
\hline & & 4 & $3,32 \pm 0,02^{b, c}$ & $1,2 \pm 0,6$ & $31,8 \pm 0,5$ \\
\hline
\end{tabular}

a,b,c Diferentes letras na mesma coluna significam tratamentos com diferenças significativas $(p<0,05) ;{ }^{(1)}$ Sem diferenças significativas $(p>0,05)$.

\section{Conclusões}

O medronheiro, apesar de todo o seu potencial, não é ainda convenientemente explorado, mas tem vindo a ganhar popularidade ao longo dos anos, com o aparecimento no mercado de novos produtos à base de medronho, nomeadamente polpas e sucos.

A aplicação de uma etapa de tratamento enzimático utilizando a Viscozyme ${ }^{\circledR}$ L conduz à obtenção de polpas de medronho com menor viscosidade, enquanto a Pectinex ${ }^{\circledR}$ Ultra SP-L conduz a polpas com maior teor de sólidos solúveis e um aumento muito significativo no rendimento de extração (mais 15\%), face ao rendimento obtido por métodos convencionais e sem recurso a enzimas. O tratamento enzimático de pasta macerada de medronho em condições processuais atrativas permitiu ainda a obtenção de polpas sem alteração significativa no nível dos restantes parâmetros avaliados: cor, $\mathrm{pH}$, acidez titulável e sólidos totais. Os resultados deste estudo permitem considerar com elevada expectativa a aplicação das carboidrases do tipo 
Viscozyme ${ }^{\circledR}$ L e Pectinex ${ }^{\circledR}$ Ultra SP-L U na extração de polpa de medronho de forma rentável, e a diversificação do leque de produtos provenientes do medronho, de acordo com as características pretendidas para indústria alimentar.

\section{Agradecimentos}

Os autores agradecem ao Fundo Europeu de Desenvolvimento Regional (FEDER) pelo apoio financeiro, através do programa Portugal2020 - CENTRO2020, no âmbito do projeto CENTRO-01-0145-FEDER023631 SoSValor - Soluções Sustentáveis para a Valorização de Produtos Naturais e Resíduos Industriais de Origem Vegetal e do COMPETE2020, no âmbito do projeto LISBOA-01-0247-FEDER-024524 MobFood - Mobilização de conhecimento científico e tecnológico em resposta aos desafios do mercado agroalimentar e do Projeto PRODER (Programa de Desenvolvimento Rural) Melhoramento da espécie e a valorização do Medronheiro (Medida 4.1, Cooperação para a Inovação, Ref. ${ }^{a}$ 53106, IPC / ESAC).

\section{Referências}

Alarcão-e-Silva, M. L. C. M. M., Leitão, A. E. B., Azinheira, H. G., \& Leitão, M. C. A. (2001). The Arbutus berry: Studies on its color and chemical characteristics at two mature stages. Journal of Food Composition and Analysis, 14(1), 27-35. http://dx.doi.org/10.1006/jfca.2000.0962

Baby, K. C., \& Ranganathan, T. V. (2016a). Effect of enzyme pre-treatment on extraction yield and quality of cardamom (Elettaria cardamomum maton.) volatile oil. Industrial Crops and Products, 89, 200-206. http://dx.doi.org/10.1016/j.indcrop.2016.05.017

Baby, K. C., \& Ranganathan, T. V. (2016b). Effect of enzyme pretreatment on yield and quality of fresh green chilli (Capsicum annuum L.) oleoresin and its major capsaicinoids. Biocatalysis and Agricultural Biotechnology, 7, 95-101. http://dx.doi.org/10.1016/j.bcab.2016.05.010

Boussalah, N., Boussalah, D., Cebadera-Miranda, L., Fernández-Ruiz, V., Barros, L., Ferreira, I. C. F. R., \& Mata, M. C. S. (2018). Nutrient composition of Algerian strawberry-tree fruits (Arbutus unedo L.). Fruits, 73(5), 283-297. http://dx.doi.org/10.17660/th2018/73.5.4

Cavaco, T. (2007). Caracterização química e bioquímica dos frutos de Arbutus unedo L. e de Rubus fruticosus Agg.: Contribuição para a sua valorização (Dissertação de mestrado). Faculdade de Engenharia de Recursos Naturais, Universidade do Algarve, Faro. Recuperado em 15 de junho de 2018, de http://hdl.handle.net/10400.1/218

Celikel, G., Demirsoy, L., \& Demirsoy, H. (2008). The strawberry tree (Arbutus unedo L.) selection in Turkey. Scientia Horticulturae, 118(2), 115-119. http://dx.doi.org/10.1016/j.scienta.2008.05.028

Chang, L. S., Karim, R., Sabo Mohammed, A., \& Mohd Ghazali, H. (2018). Characterization of enzyme-liquefied soursop (Annona muricata L.) puree. Lebensmittel-Wissenschaft + Technologie, 94, 40-49. http://dx.doi.org/10.1016/j.Iwt.2018.04.027

Global Facilitation Unit for Underutilized Species - GFU. (2013). Recuperado em 15 de junho de 2018, de http://www.underutilized-species.org

Gomes, F., \& Canhoto, J. M. (2009). Micropropagation of strawberry tree (Arbutus unedo L.) from adult plants. In Vitro Cellular \& Developmental Biology. Plant, 45(1), 72-82. http://dx.doi.org/10.1007/s11627-008-9164-8

Gomes, F., Simões, M., Lopes, M. L., \& Canhoto, J. M. (2010). Effect of plant growth regulators and genotype on the micropropagation of adult trees of Arbutus unedo L. (strawberry tree). New Biotechnology, 27(6), 882-892. PMid:20219713. http://dx.doi.org/10.1016/j.nbt.2010.02.009

Guerreiro, A. C., Gago, C. M., Miguel, M. G., \& Antunes, M. D. (2013). The effect of temperature and film covers on the storage ability of Arbutus unedo L. fresh fruit. Scientia Horticulturae, 159, 96-102. http://dx.doi.org/10.1016/j.scienta.2013.04.030

Loan, D. T. T., Tra, T. T. T., Nguyet, T. N. M., \& Man, L. V. V. (2017). Enzymatic extraction of star gooseberry (Phyllanthus acidus) juice with high antioxidant level. In AIP Conference Proceeding (pp. 3-10). Nova lorque. http://dx.doi.org/10.1063/1.5000200

Malheiro, R., Sá, O., Pereira, E., Aguiar, C., Baptista, P., \& Pereira, J. A. (2012). Arbutus Unedo L. leaves as source of phytochemicals with bioactive properties. Industrial Crops and Products, 37(1), 473-478. http://dx.doi.org/10.1016/j.indcrop.2011.07.023

Mendes, L., Freitas, V., Baptista, P., \& Carvalho, M. (2011). Comparative antihemolytic and radical scavenging activities of strawberry tree (Arbutus unedo L.) leaf and fruit. Food and Chemical Toxicology, 49(9), 2285-2291. PMid:21703325. http://dx.doi.org/10.1016/j.fct.2011.06.028

Molina, M., Pardo-De-Santayana, M., Aceituno, L., Morales, R., \& Tardío, J. (2011). Fruit production of strawberry tree (Arbutus unedo L.) in two Spanish forests. Forestry, 84(4), 419-429. http://dx.doi.org/10.1093/forestry/cpr031

Morales, P., Ferreira, I. C. F. R., Carvalho, A. M., Fernández-Ruiz, V., Sánchez-Mata, M. C., Cámara, M., Morales, R., \& Tardío, J. (2013). Wild edible fruits as a potential source of phytochemicals with capacity to inhibit lipid peroxidation. European Journal of Lipid Science and Technology, 115(2), 176-185. http://dx.doi.org/10.1002/ejlt.201200162 
Nguyen, C., \& Nguyen, H. (2018). The quality of Mulberry juice as affected by enzyme treatments. Beverages, 4(2), 41. http://dx.doi.org/10.3390/beverages4020041

Noronha, J. (2001). Metabolitos secundários do fruto de Arbutus unedo L. (Medronho) (Tese de doutoramento). Faculdade de Ciências e Tecnologia, Universidade Nova de Lisboa, Lisboa. Recuperado em 15 de junho de 2018, de http://hdl.handle.net/10362/10089

Novo Nordisk BioChem. (1998). Product Sheet: Pectinex® Ultra SP-L. Switzerland.

Novozymes. (2002). Product sheet: Viscozyme® L. Denmark. Recuperado em 15 de junho de 2018, de http://www.ebiosis.co.kr/Novozymes\%20Product\%20Sheet/Viscozyme\%20L.pdf

Novozymes. (2006). Fruit and vegetables application sheet: Pectinex® Ultra SP-L. Switzerland. Recuperado em 15 de junho de 2018, de http//olive_oil_processing_application_data.pdf

Novozymes. (2012). Product data sheet: Viscozyme® L. Denmark.

Oliveira, I., Baptista, P., Malheiro, R., Casal, S., Bento, A., \& Pereira, J. A. (2011). Influence of strawberry tree (Arbutus unedo L.) fruit ripening stage on chemical composition and antioxidant activity. Food Research International, 44(5), 1401-1407. http://dx.doi.org/10.1016/j.foodres.2011.02.009

Özcan, M., \& Haciseferoğullari, H. (2007). The Strawberry (Arbutus unedo L.) fruits: chemical composition, physical properties and mineral contents. Journal of Food Engineering, 78(3), 1022-1028. http://dx.doi.org/10.1016/j.jfoodeng.2005.12.014

Pedro, J. G. (1994). Carta da distribuição de figueira e medronheiro: notícia explicativa II.6 (39 p.). Lisboa: Ministério do Ambiente e Recursos Naturais, Direção Geral do Ambiente. Recuperado em 15 de junho de 2018, de

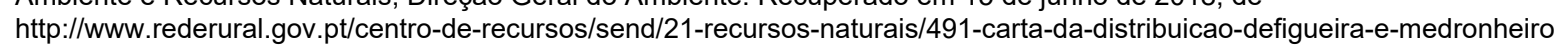

Prada, M. A., \& Arizpe, D. (2008). Riparian tree and shrub propagation handbook: An Aid to Riverine Restoration in the Mediterranean Region. Valencia: Generalitat Valenciana. Recuperado em 15 de junho de 2018, de http://www.cma.gva.es/webdoc/documento.ashx?id=143054

Rodrigues, I. M., Carvalho, M. G. V. S., \& Rocha, J. M. S. (2014). Increasing the protein content of rapeseed meal by enzymatic hydrolysis of carbohydrates. BioResources, 9(2), 2010-2025. http://dx.doi.org/10.15376/biores.9.2.2010-2025

Ruiz-Rodríguez, B.-M., Morales, P., Fernández-Ruiz, V., Sánchez-Mata, M.-C., Cámara, M., Díez-Marqués, C., Pardo-deSantayana, M., Molina, M., \& Tardío, J. (2011). Valorization of wild strawberry-tree fruits (Arbutus unedo L.) through nutritional assessment and natural production data. Food Research International, 44(5), 1244-1253. http://dx.doi.org/10.1016/j.foodres.2010.11.015

Santo, D. E., Galego, L., Gonçalves, T., \& Quintas, C. (2012). Yeast diversity in the Mediterranean strawberry tree (Arbutus unedo L.) fruits' fermentations. Food Research International, 47(1), 45-50. http://dx.doi.org/10.1016/j.foodres.2012.01.009

Siddiq, M., Dolan, K. D., Perkins-Veazie, P., \& Collins, J. K. (2018). Effect of pectinolytic and cellulytic enzymes on the physical, chemical, and antioxidant properties of blueberry (Vaccinium corymbosum L.) juice. Lebensmittel-Wissenschaft + Technologie, 92, 127-132. http://dx.doi.org/10.1016/j.Iwt.2018.02.008

StatSoft, Inc. (2013). Statistica: Electronic statistics textbook. Tulsa.

Sulusoglu, M., Cavusoglu, A., \& Erkal, S. (2011). Arbutus unedo L. (Strawberry tree) selection in Turkey Samanli mountain locations. Journal of Medicinal Plants Research, 5, 3545-3551. Recuperado em 15 de junho de 2018 , de http://www.academicjournals.org/JMPR

Torres, J. A., Valle, F., Pinto, C., Garcia-Fuentes, A., Salazar, C., \& Cano, E. (2002). Arbutus unedo L. communities in southern Iberian Peninsula mountains. Plant Ecology, 160(2), 207-223. http://dx.doi.org/10.1023/A:1015864821706

Wong, C. W., Pui, L. P., \& Ng, J. M. L. (2015). Production of spray-dried Sarawak pineapple (Ananas comosus) powder from enzyme liquefied puree. International Food Research Journal, 22(4), 1631-1636. Recuperado em 15 de junho de 2018, de http://www.ifrj.upm.edu.my/22\%20(04)\%202015/(44).pdf

Funding: FEDER (Fundo Europeu de Desenvolvimento Regional), através do programa Portugal2020 - CENTRO2020, projeto CENTRO-01-0145-FEDER-023631 SoSValor - Soluções

Sustentáveis para a Valorização de Produtos Naturais e Resíduos Industriais de Origem Vegetal e COMPETE2020, projeto LISBOA-01-0247-FEDER-024524 MobFood -

Mobilização de conhecimento científico e tecnológico em resposta aos desafios do mercado agroalimentar e Projeto PRODER (Programa de Desenvolvimento Rural) Melhoramento da espécie e a valorização do Medronheiro (Medida 4.1, Cooperação para a Inovação, Ref. ${ }^{a} 53106$, IPC/ESAC). 